\title{
Equivalent Lap Time Minimization Strategies for a Hybrid Electric Race Car
}

\section{Conference Paper}

\section{Author(s):}

Salazar Villalon, Mauro (D); Balerna, Camillo; Chisari, Eugenio; Bussi, Carlo; Onder, Christopher H.

Publication date:

2018-12

Permanent link:

https://doi.org/10.3929/ethz-b-000319994

Rights / license:

In Copyright - Non-Commercial Use Permitted

Originally published in:

https://doi.org/10.1109/CDC.2018.8618724 


\title{
Equivalent Lap Time Minimization Strategies for a Hybrid Electric Race Car
}

\author{
Mauro Salazar $^{1}$, Camillo Balerna ${ }^{1}$, Eugenio Chisari ${ }^{1}$, Carlo Bussi ${ }^{2}$, and Christopher H. Onder ${ }^{1}$
}

\begin{abstract}
The powertrain of the Formula 1 car is composed of an electrically turbocharged internal combustion engine and an electric motor used for boosting and regenerative braking. The energy management system that controls this hybrid electric power unit strongly influences the achievable lap time, as well as the fuel and battery consumption. Therefore, it is important to design robust feedback control algorithms that can run on the ECU in compliance with the sporting regulations, and are able to follow lap time optimal strategies while properly reacting to external disturbances. In this paper, we design feedback control algorithms inspired by equivalent consumption minimization strategies (ECMS) that adapt the optimal control policy implemented on the car in real-time. This way, we are able to track energy management strategies computed offline in a lap time optimal way using three PID controllers. We validate the presented control structure with numerical simulations and compare it to a previously designed model predictive control scheme.
\end{abstract}

\section{INTRODUCTION}

$\mathrm{L}$ AP TIME is of crucial importance in Formula 1 (F1). It is defined as the amount of time it takes a race car to finish a lap and it is strongly influenced by the performance of the propulsion system mounted on-board. Since 2014, F1 racing cars have been equipped with a hybrid electric powertrain, as shown in the upper part of Fig. 1. The control and coordination of such an inherently complex system is of paramount importance to achieve the best possible lap time and maximize the chances to win a race.

Herein, a 1.61 turbocharged internal combustion engine (ICE) is combined with an electric energy recovery system consisting of a battery and two electric motor/generator units (MGU). The first one is labeled kinetic, with the technical acronym MGU-K, and is connected to the traction system, to be used for electric boosting and regenerative braking. The heat unit (MGU-H) acts on the turbocharger shaft, improving the engine operation during transients and recovering waste heat from the exhaust gas. As shown in the lower part of Fig. 1, the power unit control system of the F1 car depends on the pedal operation. If the driver is not requesting full power, it is compulsory to deliver the amount of power requested, and the energy management system can only decide how to split it between the ICE and the MGU-K. Conversely, if the driver is requesting maximum power, the regulations allow the energy management system to decide the amount of propulsive power to provide and the split between the actuators. The degree of freedom offered by the thrust controller allows to trade off lap time against energy

\footnotetext{
${ }^{1}$ Institute for Dynamic Systems and Control, ETH Zürich, 8092 Zürich, Switzerland maurosalazareidsc.mavt.ethz.ch

${ }^{2}$ Power Unit Performance and Control, Ferrari S.p.A., 41053 Maranello, Italy carlo.bussieferrari.com
}

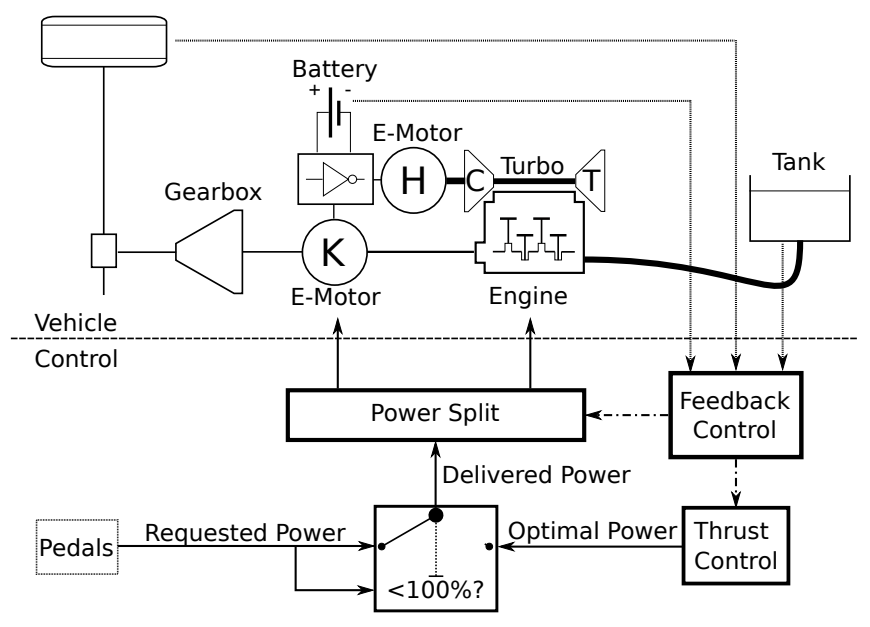

Fig. 1. The F1 power unit with the energy management feedback control system.

consumption and cope with the finite size of the battery and the fuel consumption limit of $105 \mathrm{~kg}$ per race [1]-[4].

We identify three main areas of research related to the minimum lap time energy management of hybrid electric race cars. The first one comprehends the minimum fuel control problem for hybrid electric passenger vehicles or heavy duty trucks, whereby the propulsive power is decided by the driver and the vehicle speed is treated as an exogenous signal. Researchers have studied the optimal power split problem using non-causal approaches based on dynamic programming [5], [6], convex optimization [7][9] and Pontryagin's minimum principle [10], [11]. The implementation of real-time control algorithms relied on adapting the optimal solution obtained with Pontryagin's minimum principle (PMP) [12] using equivalent consumption minimization strategies (ECMS) [13]-[17], model predictive control (MPC) algorithms [18], [19], or heuristic rulebased strategies [10], [20]. A second area of research has emerged more recently, with the introduction of adaptive cruise control systems. Under the assumption that the power unit torque, and therefore the speed of the vehicle, can be controlled, eco-driving strategies were assessed offline using dynamic programming [21], [22], nonlinear optimization [23], [24] and Pontryagin's minimum principle [25], [26], whereby the implementation in real-time was mostly based on MPC [27], [28]. Nevertheless, such approaches aim at minimizing the fuel consumption and sometimes also pollutant emissions as well as battery wearing, but never solve minimum time control problems.

A third area of research optimizes the velocity profile 
simultaneously with the driving path of race cars and motorbikes [29]-[31], occasionally including the energy recovery system [32], using nonlinear optimization techniques which result in long computational times and lack global optimality guarantees.

In our previous work, we assumed that the driving path cannot be influenced by the energy management, but is rather determined by the driver's steering input and is therefore fixed. We solved the optimal energy management problem numerically and analytically using convex optimization and PMP [33], [34]. Doing so, we provide an effective way to implement the globally optimal control strategies on the car in a feedforward fashion that can be easily tuned [35]. To introduce feedback, we designed a two-level MPC scheme which was shown to react in a lap time optimal way to realistic disturbances, while delivering satisfying results over multiple laps [36]. However, such an approach holds the drawback that optimization algorithms must run on-board, which is not always possible for an ECU.

In this paper, we present a feedback control approach which achieves a better performance than the presented MPC algorithms, while showing a negligible computational effort. Leveraging techniques inspired by ECMS, we propose equivalent lap time minimization strategies to adapt the optimal power split and thrust control strategies using PID controllers, in order to react to disturbances and respect the consumption limits in a lap time optimal way.

The structure of this paper is as follows: Section II presents a convex model of the F1 car, focusing on its hybrid electric powertrain. Thereafter, we design equivalent lap time minimization strategies to optimally track precomputed nominal trajectories. Section III compares the results obtained with the presented controller and with the previously developed MPC scheme on a benchmark simulator. Furthermore, we provide a comparison to the globally optimal solution. Section IV concludes the paper.

\section{Methodology}

This section describes the F1 powertrain and the sporting regulations. Subsequently, we derive equivalent lap time minimization strategies for the feedback control of the hybrid electric power unit.

The lower part of Fig. 1 shows that the pedal operation influences the capabilities of the F1 energy management system. If the driver is at full throttle, i.e., she/he is requesting maximum thrust, the car is in a power-limited region: Its performance is limited by the amount of thrust that can be provided by the powertrain. Such a scenario happens on the straights, between the exit of one corner and the next braking point. In order to respect the limitations on fuel and battery usage, it is permitted to overwrite this $100 \%$ power request and use a thrust controller: The amount of power to be delivered by the ICE and the MGU-K can be chosen independently. Conversely, if the driver is not requiring maximum power, the car is in a grip-limited region, as its speed is limited by the adherence of the tires on the lane and a larger thrust than requested may result in a dangerous drift. In this case, the power request must be met and we can only decide how to split it between the ICE and the MGU-K [3]. In this paper, we reformulate the optimal control

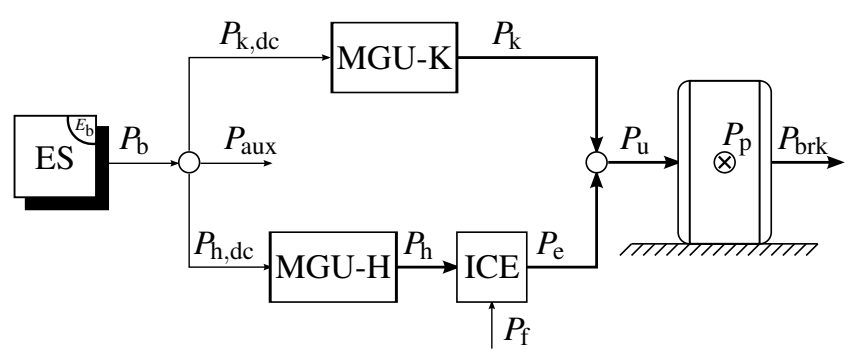

Fig. 2. The F1 power unit.

policy derived in [34], which can be implemented on the ECU in compliance with the regulations, for the power split and the thrust control, and adapt it in real-time.

\section{A. Longitudinal Dynamics and Powertrain Model}

This section summarizes the model of the F1 car shown in Fig. 2. Detailed information is to be found in [33]. We represent the car as a point with mass $m$ at the longitudinal position $s$ and moving with speed $v$. Its kinetic energy $E_{\text {kin }}=\frac{m}{2} \cdot v^{2}$ is subject to propulsive power $P_{\mathrm{p}}$ and drag power $P_{\mathrm{d}}$ as

$$
\frac{\mathrm{d}}{\mathrm{d} t} E_{\mathrm{kin}}(t)=P_{\mathrm{p}}(t)-P_{\mathrm{d}}(t) .
$$

The propulsive power is related to the power delivered by the power unit $P_{\mathrm{u}}$ and the power extracted by the hydraulic brakes $P_{\text {brk }}$ as

$$
P_{\mathrm{p}}=c_{\mathrm{s}, 1} \cdot P_{\mathrm{u}}^{2}+c_{\mathrm{s}, 2} \cdot P_{\mathrm{u}}-P_{\mathrm{brk}},
$$

where the coefficients $c_{\mathrm{s}, i}$ represent the wheels' slip. The drag power is a sum of aerodynamic drag, rolling friction and gravitational power. We condense the vehicle dynamics, the shape of the path on the track and the acceleration constraints into a position-dependent maximum kinetic energy constraint as

$$
E_{\mathrm{kin}}(t) \leq E_{\mathrm{kin}, \max }(s(t)) .
$$

The power delivered by the power unit $P_{\mathrm{u}}$ is a combination of the mechanical power delivered by the ICE $P_{\mathrm{e}}$ and by the MGU-K $P_{\mathrm{k}}$. The former is related to the fuel power $P_{\mathrm{f}}$ by the Willans approximation $P_{\mathrm{e}}=\eta_{\mathrm{e}} \cdot P_{\mathrm{f}}-P_{\mathrm{e}, 0}$, where $\eta_{e}$ and $P_{\mathrm{e}, 0}$ represent the internal efficiency of the ICE and its drag power, respectively [37]. The fuel flow limitations are expressed as

$$
0 \leq P_{\mathrm{f}} \leq P_{\mathrm{f}, \max } .
$$

The MGU-K electrical power is limited to $120 \mathrm{~kW}$ for both motor and generator operating modes. We express this constraint for the mechanical power as

$$
P_{\mathrm{k}, \min } \leq P_{\mathrm{k}} \leq P_{\mathrm{k}, \max } .
$$

Assuming a quasi-static operation, the MGU-H is modeled as a generator extracting the mechanical power $P_{\mathrm{h}}=-\eta_{\mathrm{h}} \cdot P_{\mathrm{f}}$, where $\eta_{\mathrm{h}}$ is the efficiency of the turbocompound system. The electrical power of the MGUs $P_{\mathrm{x}, \mathrm{dc}}$, with $\mathrm{x} \in\{\mathrm{k}, \mathrm{h}\}$, is modeled as

$$
P_{\mathrm{x}, \mathrm{dc}}=\alpha_{\mathrm{x}} \cdot P_{\mathrm{x}}^{2}+P_{\mathrm{x}}
$$


where the coefficients $\alpha_{\mathrm{x}}$ quantify the power losses. The power extracted at the battery terminal $P_{\mathrm{b}}$ is the sum of the MGU-K and MGU-H electrical power and of the constant auxiliary power $P_{\text {aux }}=2 \mathrm{~kW}$, that is

$$
P_{\mathrm{b}}=P_{\mathrm{aux}}+P_{\mathrm{k}, \mathrm{dc}}+P_{\mathrm{h}, \mathrm{dc}}
$$

We model the internal battery power $P_{\mathrm{i}}$ as in (6) as a function of the terminal battery power:

$$
P_{\mathrm{i}}=\alpha_{\mathrm{b}} \cdot P_{\mathrm{b}}^{2}+P_{\mathrm{b}},
$$

where $\alpha_{\mathrm{b}}$ is a coefficient approximating the losses of the battery internal resistance.

The state variables of the powertrain are the fuel energy consumption $E_{\mathrm{f}}$ and the battery state of energy $E_{\mathrm{b}}$, which are modeled as open integrators:

$$
\begin{aligned}
& \frac{\mathrm{d}}{\mathrm{d} t} E_{\mathrm{f}}(t)=P_{\mathrm{f}}(t) \\
& \frac{\mathrm{d}}{\mathrm{d} t} E_{\mathrm{b}}(t)=-P_{\mathrm{i}}(t),
\end{aligned}
$$

where the negative sign in the second equation is due to the fact that the battery discharges when its internal power $P_{\mathrm{i}}$ is positive.

Owing to sporting regulations, there are specific limitations to take into account. During a race, the amount of regenerative braking energy transferred directly from the MGU-K to the battery is limited to $-2 \mathrm{MJ}$ per lap. This energy is labeled $E_{\mathrm{K} 2 \mathrm{ES}}$ and its dynamics are modeled as

$$
\frac{\mathrm{d}}{\mathrm{d} t} E_{\mathrm{K} 2 \mathrm{ES}}(t)=\min \left\{0, P_{\mathrm{k}, \mathrm{dc}}(t)\right\}=: P_{\mathrm{K} 2 \mathrm{ES}}(t) .
$$

\section{B. Energy Management Problem for One Race Lap}

In this section, we formulate the energy management problem for the F1 car over one race lap. The main goal is to minimize the lap time $T$, i.e.,

$$
\min \int_{0}^{T} \mathrm{~d} t
$$

subject to the dynamics and the constraints presented in the previous Section II-A and to the following terminal constraints. The limit on regenerative braking energy is expressed as

$$
E_{\mathrm{K} 2 \mathrm{ES}}(T) \geq-2 \mathrm{MJ}
$$

The finite size of the battery limits its usage only if its state of charge is close to its upper or lower limit, but there are no constraints in a single lap. Similarly, the fuel consumption is limited over the whole race only. In fact, consumption limits are used as strategy parameters and are varied depending on the scenario. This way we can define nominal fuel and battery usage per lap as $\Delta E_{\mathrm{f}, 0}$ and $\Delta E_{\mathrm{b}, 0}$, respectively, and include them in the optimization problem as

$$
\begin{aligned}
& E_{\mathrm{f}}(T)-E_{\mathrm{f}}(0) \leq \Delta E_{\mathrm{f}, 0} \\
& E_{\mathrm{b}}(T)-E_{\mathrm{b}}(0) \geq \Delta E_{\mathrm{b}, 0} .
\end{aligned}
$$
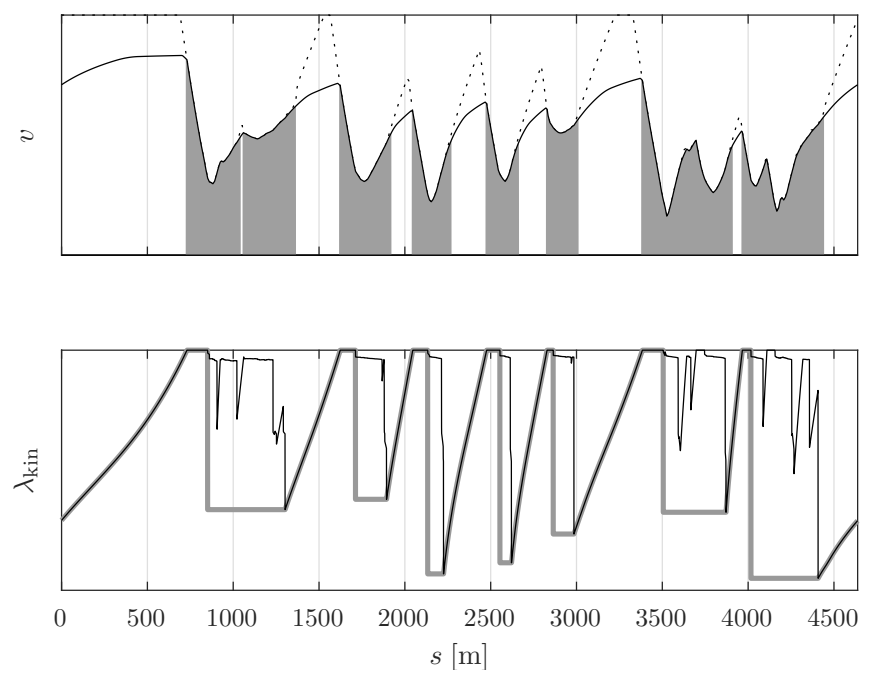

Fig. 3. Maximum (dotted) and optimal speed profile together with the power-limited (white) and the grip-limited (grey) regions. Nominal (black) and robustified (grey) kinetic costate variable $\lambda_{\text {kin }}$.

\section{Minimum Lap Time Control Policy}

In [34] we derived the minimum lap time control policy using PMP. Here, we focus on race scenarios. Specifically, we define the state variables as kinetic, fuel, battery and $\mathrm{K} 2 \mathrm{ES}$ energy $x=\left(E_{\mathrm{kin}}, E_{\mathrm{f}}, E_{\mathrm{b}}, E_{\mathrm{K} 2 \mathrm{ES}}\right)^{\top} \in \mathbb{R}^{4}$, and the input variables as ICE and MGU-K mechanical power $u=$ $\left(P_{\mathrm{e}}, P_{\mathrm{k}}\right)^{\top} \in \mathbb{R}^{2}$. The Hamiltonian of the minimum lap time control problem is then

$$
H(x, u, \lambda)=1+\lambda^{\top} \cdot f(x, u)+\Psi_{\mathscr{X} \times \mathscr{U}}(x, u),
$$

where $\lambda=\left(\lambda_{\text {kin }}, \lambda_{\mathrm{f}}, \lambda_{\mathrm{b}}, \lambda_{\mathrm{K} 2 \mathrm{ES}}\right)^{\top}$ are the costate variables, the function $f(x, u)$ describes the system dynamics presented in Section II-A, and the non-smooth function $\Psi_{\mathscr{X} \times \mathscr{U}}(x, u)$ is an indicator function on the state and input constraint sets $\mathscr{X}$ and $\mathscr{U}^{1}$ [38]. Defining the control Hamiltonian $\tilde{H}(u, \lambda)$, whereby only the input-dependent components of the Hamiltonian (14) are given, we obtain

$$
\begin{aligned}
\tilde{H}(u, \lambda):= & \lambda_{\text {kin }} \cdot P_{\mathrm{p}}(u)+\lambda_{\mathrm{f}} \cdot P_{\mathrm{f}}(u)-\lambda_{\mathrm{b}} \cdot P_{\mathrm{i}}(u) \\
& +\lambda_{\mathrm{K} 2 \mathrm{ES}} \cdot P_{\mathrm{K} 2 \mathrm{ES}}(u)+\Psi_{\mathscr{U}}(u),
\end{aligned}
$$

which is non-smooth (due to $P_{\mathrm{K} 2 \mathrm{ES}}(u)$ and $\Psi_{\mathscr{U}}(u)$ ) but convex with respect to $u$. Therefore, the optimal control policy $u^{*}$ can be obtained solving the system of inclusions $0 \in \partial_{u} \tilde{H}\left(u^{*}, \lambda\right)$ for $u^{*}$, where $\partial_{u}(\cdot)$ represents the subdifferential operator [38]. The resulting control policy is a sole function of the costate variables $\lambda$. These variables represent the sensitivity of lap time to a perturbation of the corresponding state variables and can be obtained from the numerical solution. Accordingly, the values of $\lambda_{\mathrm{f}}, \lambda_{\mathrm{b}}$ and $\lambda_{\mathrm{K} 2 \mathrm{ES}}$ represent the sensitivity of the cost function to a change in the consumption of fuel, battery and K2ES energy, respectively, and are shown to be constant over the whole lap. The kinetic costate variable $\lambda_{\text {kin }}(s)$ denotes how much lap time is affected by a change in speed depending

\footnotetext{
${ }^{1}$ This function is zero if its arguments are inside the constraint sets and infinity otherwise, and can be interpreted as a cost-based formulation of hard constraints.
} 

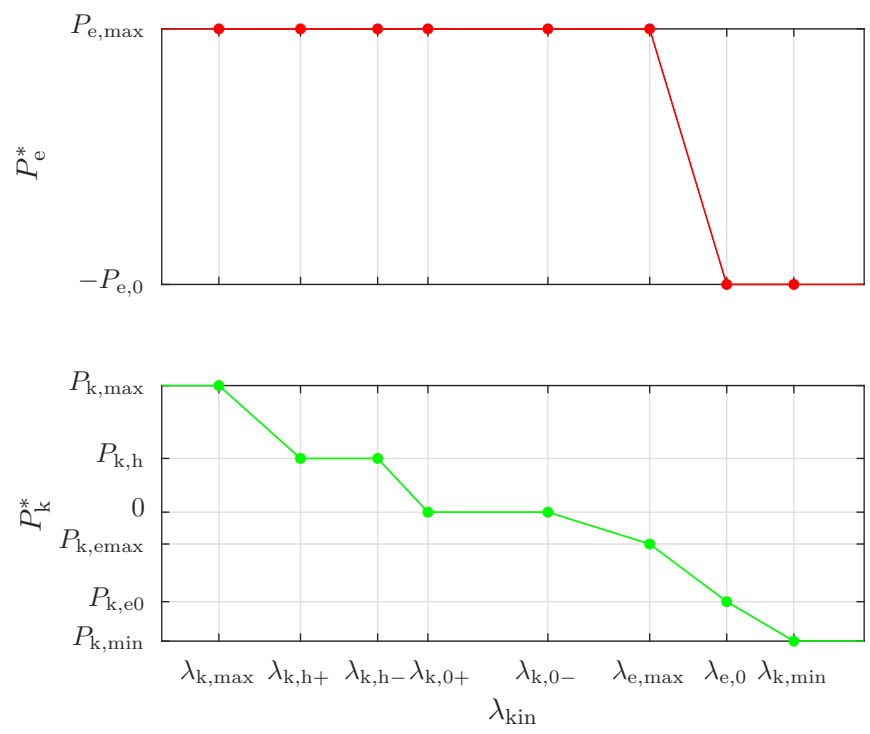

Fig. 4. Optimal ICE and MGU-K power as a function of the kinetic costate variable.

on the position on the track. Therefore, it indicates where it is most efficient to maximize the thrust. It is the only non-constant parameter in the optimal control policy. A typical trajectory of this variable is shown in Fig. 3 for the circuit of Barcelona. It abruptly falls to local minima where the maximum speed constraint becomes inactive, i.e., at the beginning of a straight. There, an increased speed would most effectively reduce lap time. The trajectory of $\lambda_{\text {kin }}$ reaches 0 in the braking points, where local changes in kinetic energy would not affect optimality. Moreover, we can distinguish two regions in the upper plot: First, the power-limited region where the maximum speed constraint is inactive and therefore the driver requires maximum thrust. In this region, according to the rules, the power request can be overwritten and the ICE and MGU-K output can be chosen independently. Second, the grip-limited region, where the driver follows the maximum speed profile attainable in the corners by requiring a precise amount of propulsive power, which must be delivered.

We treat the constant costate variables $\lambda_{\mathrm{f}}, \lambda_{\mathrm{b}}$ and $\lambda_{\mathrm{K} 2 \mathrm{ES}}$ as given parameters of the optimal control policy. We compute the optimal ICE and MGU-K power as functions of the kinetic costate variable as

$$
\begin{aligned}
& P_{\mathrm{e}}^{*}\left(\lambda_{\text {kin }}\right) \\
& P_{\mathrm{k}}^{*}\left(\lambda_{\text {kin }}\right),
\end{aligned}
$$

which are shown in Fig. 4. Since these functions are piecewise affine, they can be evaluated with first-order interpolations on the given thresholds. They are suited for the powerlimited regions, since there the ICE and MGU-K power can be decided independently. Nevertheless, in the grip-limited regions, where the power request must be fulfilled, we use the optimal power split

$$
\left[P_{\mathrm{e}}^{*}, P_{\mathrm{k}}^{*}\right]=\operatorname{PS}\left(P_{\text {req }}\right) \text {, }
$$

which can be obtained by applying PMP to the same problem, where the power to be delivered is given. The control

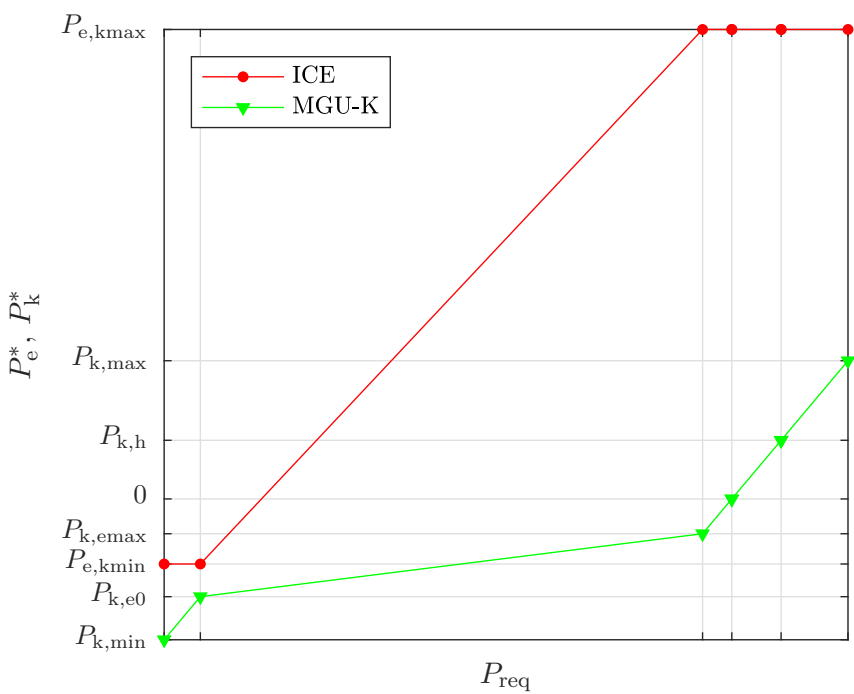

Fig. 5. Optimal power split: Optimal ICE and MGU-K power as a function of the total power unit power.

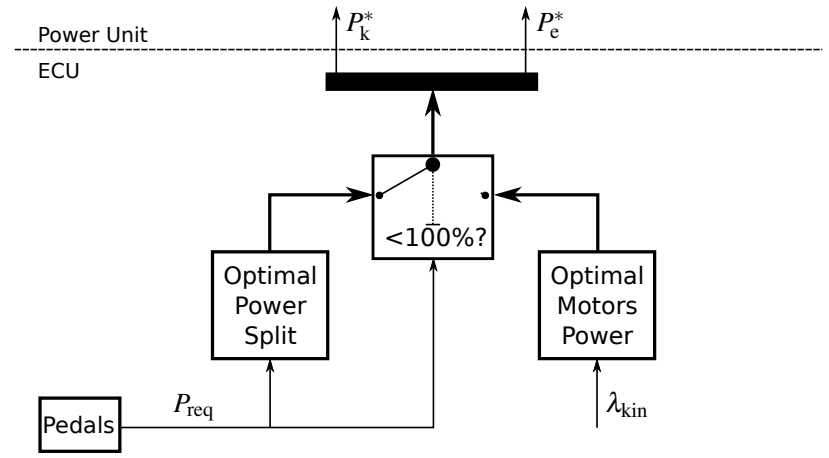

Fig. 6. Optimal control policy (OCP) implementation. The look-up tables for the optimal ICE and MGU-K power and the optimal power split, shown in Fig. 4 and 5, respectively, are computed using the values of $\lambda_{\mathrm{f}}, \lambda_{\mathrm{b}}$ and $\lambda_{\mathrm{K} 2 \mathrm{ES}}$.

Hamiltonian in that case would correspond to (15) without the term $\lambda_{\text {kin }} \cdot P_{\mathrm{p}}(u)$, but with the additional constraint $P_{\mathrm{u}}=$ $P_{\text {req. }}$. Fig. 5 shows the corresponding piecewise affine optimal power split as a function of $P_{\text {req. }}$.

The optimal control policy can be implemented compatibly with the regulations as shown in Fig. 6 by using the optimal motors power (16) in the power-limited region and the optimal power split (17) in the grip-limited region.

\section{Equivalent Lap Time Minimization Strategies}

The optimal control policy presented in the previous Section II-C and shown in Fig. 6 can be implemented in a feedforward fashion as follows: We solve the numerical optimal control problem presented in Section II-A offline employing a dedicated second-order conic program solver [33]. We store the costate variables $\lambda$ obtained, whereby we use the kinetic costate variable $\lambda_{\text {kin }}(s)$ as a decision variable in the power-limited region, and the constant costate values of $\lambda_{\mathrm{f}}, \lambda_{\mathrm{b}}$ and $\lambda_{\mathrm{K} 2 \mathrm{ES}}$ serve to evaluate the optimal ICE and MGU-K power (16) and the optimal power split (17) to be 


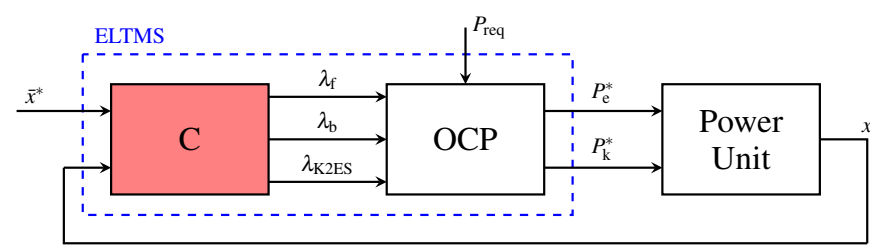

Fig. 7. Structure of the equivalent lap time minimization strategies (ELTMS). A controller is responsible for tracking the nominal state trajectories $\bar{x}^{*}$ by adapting the costate variables, which determine the optimal control policy (OCP) shown in Fig. 6 responsible to require the ICE and MGU-K power to the powertrain low-level control system.

used in real-time. This approach works well under nominal conditions, i.e., no model uncertainty and no disturbances. However, for the sake of robustness, a feedback on the energy management is necessary to avoid dangerous drifts in fuel and battery consumption, and to follow precomputed optimal strategies.

In this section, we present an ECMS-inspired approach, namely, equivalent lap time minimization strategies (ELTMS), whereby we adapt the optimal control policy in real-time by controlling the costate variables.

The optimal trajectory of the kinetic costate variable $\lambda_{\text {kin }}(s)$ is not significantly affected by changes in energy budgets, i.e., the values $\Delta E_{\mathrm{f}, 0}$ and $\Delta E_{\mathrm{b}, 0}$ chosen for a specific lap [35]. Therefore, we do not control its trajectory but use it as a given exogenous variable. Nevertheless, a robustification is necessary. If exiting a corner the driver would go at full throttle earlier than expected, the car would be in a powerlimited region where the kinetic costate variable is almost zero. Accordingly, very little power would be deployed at the beginning of the subsequent straight with a consequent nonnegligible lap time loss. Driven by this rationale, we modify the trajectory of the kinetic costate variable in the nominal grip limited regions as follows: As it is possible to see in Fig. 3, between the apex of a corner, coinciding with the local minimum of the speed profile, and the end of the griplimited region containing it, the value of the kinetic costate variable is set equal to its value at the beginning of the next power-limited region. This way, if the driver was to require maximum power earlier than expected, the powertrain would deploy the same amount of power as at the beginning of the power-limited region under nominal conditions. Conversely, no effect would be noticed if the driver required less than $100 \%$ power, since then such a power request would be met, ignoring the value of the kinetic costate variable $\lambda_{\text {kin }}$.

Considering the kinetic costate trajectory as given, the only parameters affecting the optimal control policy shown in Fig. 6 are the constant costate variables related to fuel, battery and K2ES consumption $\lambda_{\mathrm{f}}, \lambda_{\mathrm{b}}$ and $\lambda_{\mathrm{K} 2 \mathrm{ES}}$, respectively. Therefore, we design ELTMS to adapt these costate variables, in order to track precomputed nominal state trajectories $\bar{x}^{*}$, as shown in Fig. 7. An intuitive approach would be to build three SISO control loops or a unique MIMO controller, relating each costate to its corresponding state variable. However, such an approach did not work due to the non-smooth nature of the energy management: In the griplimited region, there is only one degree of freedom, namely the power split, and it is possible to decide how to trade fuel with battery charge. In the power-limited region, the additional degree of freedom granted by the thrust control allows to trade lap time for energy consumption.

Motivated by such a rationale, and similarly to multivariable ECMS [17], we reformulate the costate variables. First, we define a lap time equivalence factor $q_{\mathrm{t}} \geq 0$, and the fuel-to-electricity weight $q_{\mathrm{f}} \in[0,1]$ and the K2ES-to-battery weight $q_{\mathrm{K} 2 \mathrm{ES}} \in[0,1]$. Then, we express the fuel, battery and $\mathrm{K} 2 \mathrm{ES}$ costate variables as

$$
\begin{aligned}
& \lambda_{\mathrm{f}}=q_{\mathrm{t}} \cdot q_{\mathrm{f}} \\
& \lambda_{\mathrm{b}}=-q_{\mathrm{t}} \cdot\left(1-q_{\mathrm{f}}\right) \cdot\left(1-q_{\mathrm{K} 2 \mathrm{ES}}\right) \\
& \lambda_{\mathrm{K} 2 \mathrm{ES}}=-q_{\mathrm{t}} \cdot\left(1-q_{\mathrm{f}}\right) \cdot q_{\mathrm{K} 2 \mathrm{ES}},
\end{aligned}
$$

and substitute them in the control Hamiltonian (15) as

$$
\begin{aligned}
\tilde{H}(u, \lambda):= & \lambda_{\text {kin }} \cdot P_{\mathrm{p}}(u)+q_{\mathrm{t}} \cdot\left(q_{\mathrm{f}} \cdot P_{\mathrm{f}}(u)\right. \\
& +\left(1-q_{\mathrm{f}}\right) \cdot\left(\left(1-q_{\mathrm{K} 2 \mathrm{ES}}\right) \cdot P_{\mathrm{i}}(u)\right. \\
& \left.\left.-q_{\mathrm{K} 2 \mathrm{ES}} \cdot P_{\mathrm{K} 2 \mathrm{ES}}(u)\right)\right)+\Psi_{\mathscr{U}}(u) .
\end{aligned}
$$

Defining the weighted consumption power as

$$
\begin{gathered}
P_{\mathrm{c}}\left(u, q_{\mathrm{f}}, q_{\mathrm{K} 2 \mathrm{ES}}\right)=q_{\mathrm{f}} \cdot P_{\mathrm{f}}(u)+\left(1-q_{\mathrm{f}}\right) \cdot\left(\left(1-q_{\mathrm{K} 2 \mathrm{ES}}\right) \cdot P_{\mathrm{i}}(u)\right. \\
\left.-q_{\mathrm{K} 2 \mathrm{ES}} \cdot P_{\mathrm{K} 2 \mathrm{ES}}(u)\right),
\end{gathered}
$$

the control Hamiltonian (19) can be expressed as

$$
\tilde{H}(u, \lambda)=\lambda_{\text {kin }} \cdot P_{\mathrm{p}}(u)+q_{\mathrm{t}} \cdot P_{\mathrm{c}}\left(u, q_{\mathrm{f}}, q_{\mathrm{K} 2 \mathrm{ES}}\right)+\Psi_{\mathscr{U}}(u) .
$$

At this point, some observations can be made. First, the control Hamiltonian (21) shows that the equivalence factor $q_{\mathrm{t}}$ defines the trade-off between lap time and consumption. In fact, it affects only the power-limited regions, where the kinetic costate variable $\lambda_{\text {kin }}$ is used to determine how much power to provide with both the ICE and the MGU-K as shown in Fig. 4. In the grip-limited regions, the amount of power to be delivered is determined by the driver and the equivalence factor $q_{\mathrm{t}}$ has no impact on the power split shown in Fig. 5: The trade-off between fuel and electric energy consumption is solely determined by the weights $q_{\mathrm{f}}$ and $q_{\mathrm{K} 2 \mathrm{ES}}$. Second, deviations in fuel and K2ES energy profiles are to be expected only in the grip-limited regions, since in the straights the ICE is almost always operated at full power and the MGU-K is not used in generator mode, i.e., by braking the ICE. These observations allow us to define the control loops as follows: We use the equivalence factor $q_{\mathrm{t}}$ in the power-limited regions to control the weighted energy consumption

$$
\begin{aligned}
& E_{\mathrm{c}}\left(E_{\mathrm{f}}, E_{\mathrm{b}}, E_{\mathrm{K} 2 \mathrm{ES}}, q_{\mathrm{f}}, q_{\mathrm{K} 2 \mathrm{ES}}\right)=q_{\mathrm{f}} \cdot E_{\mathrm{f}} \\
& \quad-\left(1-q_{\mathrm{f}}\right) \cdot\left(\left(1-q_{\mathrm{K} 2 \mathrm{ES}}\right) \cdot E_{\mathrm{b}}+q_{\mathrm{K} 2 \mathrm{ES}} \cdot E_{\mathrm{K} 2 \mathrm{ES}}\right)
\end{aligned}
$$

so that it tracks its nominal trajectory precomputed offline $\bar{E}_{\mathrm{c}}^{*}=E_{\mathrm{c}}\left(\bar{E}_{\mathrm{f}}^{*}, \bar{E}_{\mathrm{b}}^{*}, \bar{E}_{\mathrm{K} 2 \mathrm{ES}}^{*}, q_{\mathrm{f}}, q_{\mathrm{K} 2 \mathrm{ES}}\right)$, where the notation $(\bar{\cdot})^{*}$ denotes the optimal trajectory under nominal conditions. On the other hand, in the grip-limited region, we use the weights $q_{\mathrm{f}}$ and $q_{\mathrm{K} 2 \mathrm{ES}}$ to control the fuel and the K2ES energy $E_{\mathrm{f}}$ and $E_{\mathrm{K} 2 \mathrm{ES}}$. These three SISO control loops can be handled with standard PID controllers, only active in the power-limited or grip-limited regions, respectively, as shown in Fig. 8. To summarize, a PID controller uses the lap time equivalence factor $q_{\mathrm{t}}$ as an actuator in power-limited regions to stabilize the deviation in energy consumption $\delta E_{\mathrm{c}}=E_{\mathrm{c}}-\bar{E}_{\mathrm{c}}^{*}$, whereas 


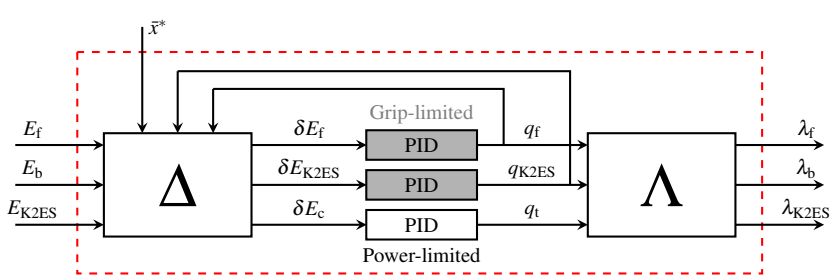

Fig. 8. Controller of the ELTMS shown in Fig. 7 for the adaptation of the costate variables. The $\Delta$-block computes the value of $E_{\mathrm{c}}$ as in (22) and the deviations of this variable, the fuel and the K2ES energy from their nominal trajectories. The PIDs of fuel and K2ES (grey) only operate in grip-limited regions, whereas the PID of the energy consumption (white) only in powerlimited regions. The $\Lambda$-block computes the variables $\left(\lambda_{\mathrm{f}}, \lambda_{\mathrm{b}}, \lambda_{\mathrm{K} 2 \mathrm{ES}}\right)$ from the values of $q_{\mathrm{t}}, q_{\mathrm{f}}$ and $q_{\mathrm{K} 2 \mathrm{ES}}$, as in eq. (18).
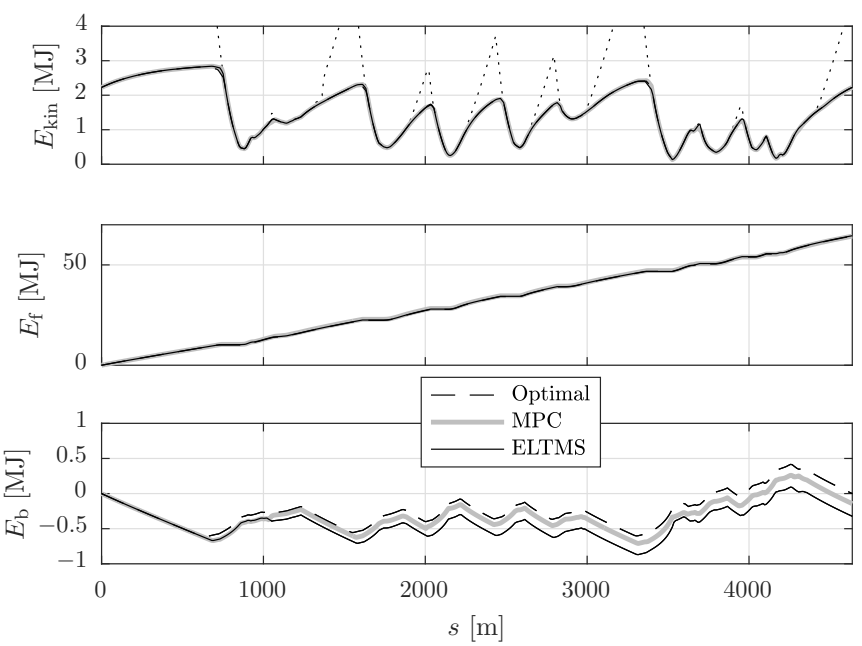

Fig. 9. State trajectories over one lap for the fresh tires scenario.

the weights $q_{\mathrm{f}}$ and $q_{\mathrm{K} 2 \mathrm{ES}}$ are used by two PID controllers in grip-limited regions to stabilize the fuel and K2ES deviations $\delta E_{\mathrm{f}}=E_{\mathrm{f}}-\bar{E}_{\mathrm{f}}^{*}$ and $\delta E_{\mathrm{K} 2 \mathrm{ES}}=E_{\mathrm{K} 2 \mathrm{ES}}-\bar{E}_{\mathrm{K} 2 \mathrm{ES}}^{*}$, respectively.

\section{RESULTS}

This section presents the results obtained by implementing the ELTMS on a benchmark simulator [34] in the presence of a realistic race disturbance. Since the simulator used is based on a convex model of the system, we can compute the globally optimal solution and the achievable lap time for every scenario [33]. We simulate the car mounting fresh tires. The enhanced grip in the turns allows the driver to corner faster than expected. This effect is simulated by increasing the maximum kinetic energy profile by $10 \%$. We compare the proposed feedback control scheme with the two-level MPC controller presented in [36] and the globally optimal solution computed numerically [33]. The feedback scheme is also tested over multiple laps. All simulations are carried out using the circuit of Barcelona, with a nominal fuel load of $100 \%$, i.e., $\Delta E_{\mathrm{f}, 0}$ chosen such that the same amount of fuel is to be used for each lap, and in charge-sustained battery operation, i.e., $\Delta E_{\mathrm{b}, 0}=0 \mathrm{MJ}$.

Fig. 9 and 10 show the state and input trajectories in the first lap. The tracking performance of the ELTMS algorithm is comparable to the one of the MPC, and both show a close-to-optimal behaviour in terms of lap time deviation
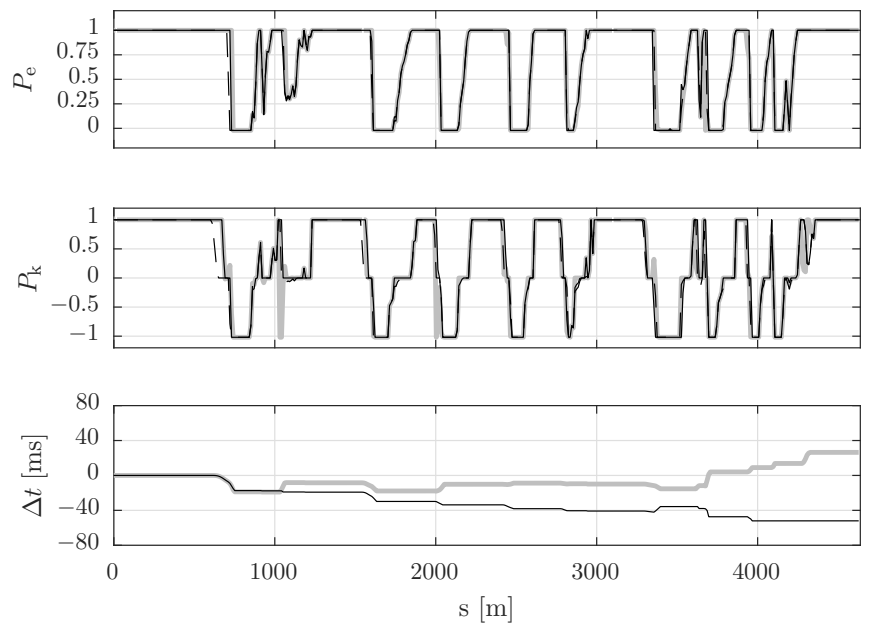

Fig. 10. Normalized input trajectories and lap time difference over one lap for the fresh tires scenario.

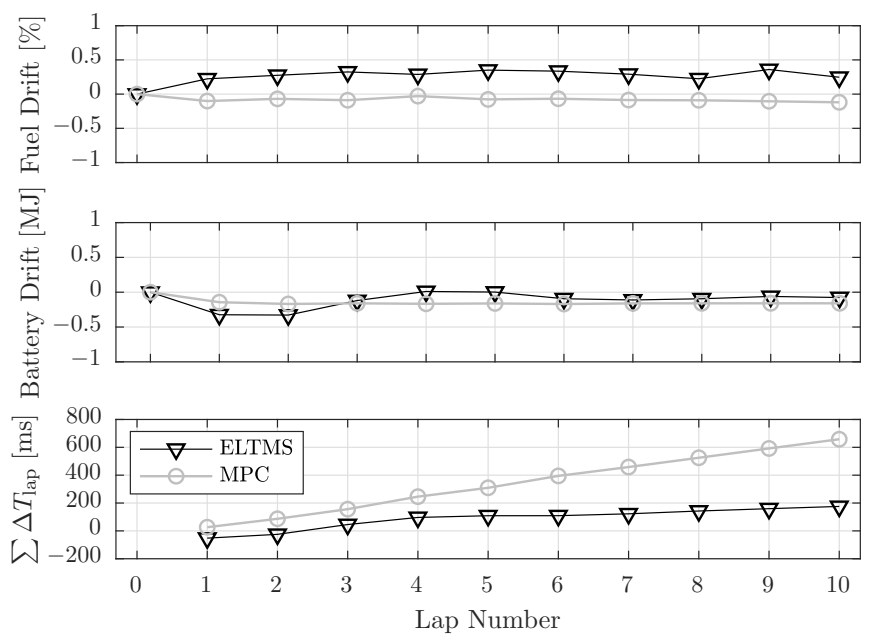

Fig. 11. Fuel, battery and cumulative lap time drift with respect to the globally optimal solution for the fresh tires scenario.

from the optimal solution. The ELTMS has a faster lap time with respect to the MPC, while drifting more in terms of battery consumption. Fig. 11, however, shows that with both algorithms the solution converges close to the desired energy budgets while avoiding dangerous drifts. Moreover, the ELTMS appears to perform better than the MPC in terms of lap time, losing less than 20 milliseconds per lap with respect to the global optimum.

\section{CONCLUSION}

In this paper, we presented equivalent lap time minimization strategies for the feedback control of the F1 hybrid electric powertrain. To take the non-smooth structure of the problem explicitly into account, we leveraged Pontryagin's minimum principle to adapt the optimal control policy in real-time with simple PID controllers.

We tested the control algorithms in the presence of a typical disturbance for a $\mathrm{F} 1$ race on a benchmark simulator. A robust and close-to-optimal behaviour was observed over one and over subsequent race laps. Moreover, the performance of the presented approach was better compared 
to the performance of a previously developed optimality tracking MPC scheme. Furthermore, it completely avoided its computational complexity.

This work opens the field for several possible extensions. First, a feedforward path could be introduced, in order to change the reference state and costate trajectories in the presence of predictable scenarios. Such scenarios comprise the diminishing mass of the vehicle due to fuel combustion, pit-stops, the presence of the safety car, and different weather conditions. Second, parameter estimation algorithms could be included, also in combination with the aforementioned feedforward approach. These algorithms could be used to adapt the model parameters during the race and thereby improve the overall control performance. Third, the controller should be extended to qualifying scenarios.

\section{ACKNOWLEDGMENTS}

We would like to thank Ferrari S.p.A. for supporting this project. Moreover, we would like to express our gratitude to Dr. Ilse New for her assistance with the proofreading, useful advice and comments. Finally, we are very thankful to Mr. Nicolas Lanzetti for helping us generate the schematic representations. Last, but not least, our gratitude also goes to Ristorante Montana for the support and the inspiration.

\section{REFERENCES}

[1] FIA, "2009 formula one technical regulations," FIA, Geneva, Switzerland, Tech. Rep., 2009.

[2] — " "2014 formula one technical regulations," FIA, Geneva, Switzerland, Tech. Rep., 2011.

[3] — " "2017 formula one technical regulations," FIA, Geneva, Switzerland, Tech. Rep., 2017.

[4] A. Picarelli and M. Dempsey, "Simulating the complete 2014 hybrid electric formula 1 cars," Hybrid and Electric Vehicles Conference (HEVC), November 2014.

[5] L. V. Pérez and E. A. Pilotta, "Optimal power split in a hybrid electric vehicle using direct transcription of an optimal control problem," Mathematics and Computers in Simulation, vol. 79, no. 6, pp. 19591970, 2009.

[6] P. Elbert, S. Ebbesen, and L. Guzzella, "Implementation of dynamic programming for $n$-dimensional optimal control problems with final state constraints," IEEE Transactions on Control Systems Technology, vol. 21, no. 3, pp. 924-931, 2013.

[7] N. Murgovski, L. Johannesson, J. Sjöberg, and B. Egardt, "Component sizing of a plug-in hybrid electric powertrain via convex optimization," Mechatronics, vol. 22, no. 1, pp. 106-120, 2012.

[8] P. Elbert, T. Nüesch, A. Ritter, N. Murgovski, and L. Guzzella, "Engine on/off control for the energy management of a serial hybrid electric bus via convex optimization," IEEE Transactions on Vehicular Technology, vol. 63, no. 8, pp. 3549-3559, 2014.

[9] T. Nüesch, P. Elbert, M. Flankl, C. Onder, and L. Guzzella, "Convex optimization for the energy management of hybrid electric vehicles considering engine start and gearshift costs," Energies, vol. 7, no. 2, pp. 834-856, 2014.

[10] L. Guzzella and A. Sciarretta, Vehicle propulsion systems, 3rd ed. Berlin: Springer, 2013.

[11] A. Sciarretta and L. Guzzella, "Control of hybrid electric vehicles," Control systems, IEEE, vol. 27, no. 2, pp. 60-70, 2007.

[12] L. S. Pontryagin, Mathematical theory of optimal processes. CRC Press, 1987.

[13] G. Paganelli, S. Delprat, T.-M. Guerra, J. Rimaux, and J.-J. Santin, "Equivalent consumption minimization strategy for parallel hybrid powertrains," in Vehicular Technology Conference, 2002. VTC Spring 2002. IEEE 55th, vol. 4. IEEE, 2002, pp. 2076-2081.

[14] L. Serrao, S. Onori, and G. Rizzoni, "A comparative analysis of energy management strategies for hybrid electric vehicles," Journal of Dynamic Systems, Measurement, and Control, vol. 133, no. 3, p. $031012,2011$.
[15] H. Pham, P. Van Den Bosch, J. Kessels, and R. Huisman, "Integrated energy and thermal management for hybrid electric heavy duty trucks," in Vehicle Power and Propulsion Conference (VPPC), 2012 IEEE. IEEE, 2012, pp. 932-937.

[16] S. Ebbesen, P. Elbert, and L. Guzzella, "Battery state-of-health perceptive energy management for hybrid electric vehicles," IEEE Transactions on Vehicular technology, vol. 61, no. 7, pp. 2893-2900, 2012.

[17] T. Nüesch, A. Cerofolini, G. Mancini, N. Cavina, C. Onder, and L. Guzzella, "Equivalent consumption minimization strategy for the control of real driving nox emissions of a diesel hybrid electric vehicle," Energies, vol. 7, no. 5, pp. 3148-3178, 2014.

[18] H. A. Borhan, A. Vahidi, A. M. Phillips, M. L. Kuang, and I. V. Kolmanovsky, "Predictive energy management of a power-split hybrid electric vehicle," in American Control Conference, 2009. ACC'09. IEEE, 2009, pp. 3970-3976.

[19] H. Borhan, A. Vahidi, A. M. Phillips, M. L. Kuang, I. V. Kolmanovsky, and S. Di Cairano, "Mpc-based energy management of a powersplit hybrid electric vehicle," IEEE Transactions on Control Systems Technology, vol. 20, no. 3, pp. 593-603, 2012.

[20] T. Hofman, M. Steinbuch, R. Van Druten, and A. Serrarens, "Rulebased energy management strategies for hybrid vehicles," International Journal of Electric and Hybrid Vehicles, vol. 1, no. 1, pp. 71-94, 2007.

[21] J. Hooker, "Optimal driving for single-vehicle fuel economy," Transportation Research Part A: General, vol. 22, no. 3, pp. 183-201, 1988.

[22] G. Heppeler, M. Sonntag, and O. Sawodny, "Fuel efficiency analysis for simultaneous optimization of the velocity trajectory and the energy management in hybrid electric vehicles," IFAC Proceedings Volumes, vol. 47, no. 3, pp. 6612-6617, 2014.

[23] Y. Saboohi and H. Farzaneh, "Model for developing an eco-driving strategy of a passenger vehicle based on the least fuel consumption," Applied Energy, vol. 86, no. 10, pp. 1925-1932, 2009.

[24] T. van Keulen, B. de Jager, D. Foster, and M. Steinbuch, "Velocity trajectory optimization in hybrid electric trucks," in American Control Conference (ACC), 2010. IEEE, 2010, pp. 5074-5079.

[25] E. Hellström, J. Åslund, and L. Nielsen, "Management of kinetic and electric energy in heavy trucks," in SAE 2010 World Congress \& Exhibition, April 2010, Detroit, MI, USA, Session: Transmission and Driveline: Hybrid. SAE International, 2010, pp. 1152-1163.

[26] A. Sciarretta, G. De Nunzio, and L. L. Ojeda, "Optimal ecodriving control: Energy-efficient driving of road vehicles as an optimal control problem," Control Systems, IEEE, vol. 35, no. 5, pp. 71-90, 2015.

[27] B. Asadi and A. Vahidi, "Predictive cruise control: Utilizing upcoming traffic signal information for improving fuel economy and reducing trip time," IEEE transactions on control systems technology, vol. 19, no. 3, pp. 707-714, 2011.

[28] L. Johannesson, N. Murgovski, E. Jonasson, J. Hellgren, and B. Egardt, "Predictive energy management of hybrid long-haul trucks," Control Engineering Practice, vol. 41, pp. 83-97, 2015.

[29] D. Casanova, "On minimum time vehicle manoeuvring: The theoretical optimal lap," Ph.D. dissertation, Cranfield University, School of Mechanical Engineering, November 2000.

[30] R. Lot and S. Evangelou, "Lap time optimization of a sports series hybrid electric vehicle," in 2013 World Congress on Engineering, 2013, pp. 1-6.

[31] J. Hauser and A. Saccon, "Motorcycle modeling for high-performance maneuvering," Control Systems, IEEE, vol. 26, no. 5, pp. 89-105, 2006.

[32] D. J. Limebeer and A. V. Rao, "Faster, higher and greener: Vehicular optimal control," IEEE Control Systems Magazine, pp. 36-56, April 2015.

[33] S. Ebbesen, M. Salazar, P. Elbert, C. Bussi, and C. H. Onder, "Time-optimal control strategies for a hybrid electric race car," IEEE Transactions on Control Systems Technology, vol. 26, no. 1, pp. 233247, 2018.

[34] M. Salazar, P. Elbert, S. Ebbesen, C. Bussi, and C. H. Onder, "Time-optimal control policy for a hybrid electric race car," IEEE Transactions on Control Systems Technology, vol. 25, no. 6, pp. 19211934, 2017.

[35] M. Salazar, C. Bussi, F. P. Grando, and C. H. Onder, "Optimal control policy tuning and implementation for a hybrid electric race car," IFACPapersOnLine, vol. 49, no. 11, pp. 147-152, 2016.

[36] M. Salazar, C. Balerna, P. Elbert, F. P. Grando, and C. H. Onder, "Real-time control algorithms for a hybrid electric race car using a two-level model predictive control scheme," IEEE Transactions on Vehicular Technology, vol. 66, no. 12, pp. 10911-10922, 2017.

[37] L. Guzzella and C. H. Onder, Introduction to modeling and control of internal combustion engine systems, 2nd ed. Berlin: Springer, 2010.

[38] R. T. Rockafellar, Convex analysis. Princeton University Press, 1997. 